\title{
CONTROLE DE DOENÇAS NÃO TRANSMISSÍVEIS - CONTEXTO HISTÓRICO E ELEMENTOS PARA SUA DISCUSSÃO
}

Control of noncommunicable diseases - historical context and elements for its discussion

${ }^{1}$ Universidade Federal do Rio Grande do Sul. Porto Alegre/RS, Brasil.

Autor para correspondência: Aloyzio Achutti. E-mail: achutti@gmail.com.

Recebido em: 10/03/2014. Revisado em: 30/09/2014. Aprovado em: 04/10/2014. 


\section{RESUMO}

O controle de doenças extrapola o domínio da biomedicina e da saúde de indivíduos. Ele depende de paradigmas que incluem comportamento humano, organização social, desenvolvimento sustentado e equidade. A trajetória para inclusão do controle das doenças crônicas e não transmissíveis na agenda da saúde pública pode ser objeto de uma releitura, com destaque para momentos que já fogem à memória e que podem exigir maior discussão e compreensão por parte de profissionais da saúde, políticos, empresários e população em geral.

\section{Palavras-chave}

Doenças Crônicas Não Transmissíveis; Equidade; Fatores de Risco; Objetivos do Milênio; Saúde Pública.

\section{ABSTRACT}

Disease control goes beyond the field of biomedicine and public health. It depends on paradigms that include human behavior, social organization, sustainable development, and equity. The trajectory for inclusion of the control of chronic and noncommunicable diseases in the public health agenda may be subject to a re-evaluation, particularly the historical aspects that may have been forgotten and that may require further discussion and understanding by health care professionals, politicians, entrepreneurs, and the general population.

\section{Keywords}

Equity; Millennium Development Goals; Noncommunicable Diseases; Public Health; Risk Factors. 


\section{Introdução}

A classificação dicotômica e simplista das doenças como transmissíveis (DT) e outras - caso das doenças (aparentemente) crônicas não transmissíveis (DCNT) - deve ter se originado no deslumbramento causado pela descoberta do papel etiopatogênico dos micro-organismos e de seus vetores, que possibilitou o controle da cadeia epidemiológica e a interferência na transmissão de doenças. É algo, portanto, de meados do século XIX, do tempo de Filippo Pacini, Louis Pasteur e John Snow. Esse rótulo reducionista reflete o desapontamento causado pela incompreensão de doenças nas quais não se encontravam germes e pela impotência em controlá-las, assumindo-as como fatalidades degenerativas. Restava apenas assistir os indivíduos afetados, na fase avançada da história natural, aliviando o sofrimento sem causar maiores danos.

A observação e a pesquisa levaram à identificação de fatores de risco - equivalentes aos germes nas DT -, relacionáveis com alterações fisiopatológicas características de diversas DCNT. Estes fatores de risco eram comuns a várias enfermidades que, por sua vez, se superpunham, sem fronteiras bem definidas. A suscetibilidade era variável e mais saliente em algumas famílias, levando a se suspeitar de características genéticas em algumas delas. Outras, entretanto, estavam relacionadas a fatores de risco comportamentais e hábitos de vida.

Assim como os micro-organismos foram responsabilizados pelo primeiro grupo (DT), controlado com tanto sucesso, as demais doenças poderiam ser controladas ao se reduzir a exposição aos fatores de risco. O sucesso no combate às DT, no entanto, não resultou de ação direta sobre agentes patogênicos, mas de saneamento básico, medidas de higiene, isolamento, interferência na cadeia epidemiológica de transmissão e intervenções no sistema de defesa do hospedeiro por meio de vacinas e da garantia da qualidade de resposta imunitária. Por outro lado, o comportamento humano é bem mais complexo e imprevisível do que o dos germes.

As DCNT têm sido rotuladas também como doenças provocadas pelo próprio homem (man-made diseases), como se a intervenção humana não estivesse também por trás das epidemias e do controle das DT.

Em meados do século, na medida em que as doenças infecciosas e parasitárias iam sendo controladas, as DCNT tornaram-se mais salientes. A partir de estudos populacionais, cresceu o interesse voltado para este conjunto de enfermidades, constatando-se também ser dobrado o ônus de comunidades de baixa renda e de países em desenvolvimento: acompanhando o baixo desenvolvimento humano, todos os problemas persistem, coexistem e comprometem com maior precocidade. 
Há uma grande quantidade de referências bibliográficas relacionadas com esse tema, inclusive acessíveis pela internet ${ }^{1}$.

Quando se pretende discutir o controle do comportamento e de doenças, não se pode perder de vista o contexto global - em um mundo onde a violência ainda persiste - e vale recordar as palavras do discurso de Winston Churchill, lido por sua esposa por ocasião da entrega do Prêmio Nobel de 1953²:

Desde a morte de Alfred Nobel, em 1896, entramos numa era de tumulto e tragédia. O poder do homem aumentou em todos os domínios, exceto no que concerne ao controle de si mesmo. No campo da ação, jamais os acontecimentos pareceram diminuir tão duramente a personalidade humana. Raramente na História, tantos fatos brutais dominaram tanto o pensamento. A grave questão apresenta-se a todos nós: será que nossos problemas ultrapassaram o nosso controle? Sem dúvida alguma, atravessamos um período em que isso pode ser verdadeiro. Portanto, devemos, com humildade, procurar o caminho da salvação.

\section{Passos na evolução da abordagem das DCNT}

Em todo o mundo, existe um interesse muito grande no estudo, na discussão e na busca de alternativas para controle das DCNT, provocado em parte pelo desenvolvimento científico e tecnológico e por aquele de responsabilidade política e social, mas também em razão de interesses secundários da indústria e do comércio internacionais, com efeitos positivos e outros nem tanto. Alguns nomes, entre tantos outros, devem ser citados ao menos para deixar bem claro que as modificações de paradigma e de objetivos programáticos dependem, no início, de pessoas em posições-chave, com clarividência, boa vontade, entusiasmo e competência. As instituições, embora lhes deem respaldo e autoridade, servem para estruturar e manter o que está consolidado em programas de controle.

Na perspectiva deste autor, a trajetória de inclusão das DCNT poderia ser assim traçada: (1) início (década de 1950), com assistência individual a doenças crônicas isoladas em fase avançada ou terminal; (2) desenvolvimento científico e tecnológico levando à progressiva especialização e, em contrapartida, valorização da extensão de cobertura e da atenção primária; (3) desenvolvimento da epidemiologia das doenças; (4) progressiva incorporação de DCNT isoladas na agenda de saúde pública; (5) prevenção secundária individual (identificação precoce e tentativa de retardo do dano); (6) progressos na

\footnotetext{
${ }^{1}$ Seleção de referências relacionadas com as DCNT acessíveis pela internet em AMICOR. Disponível em: <http://amicor.blogspot.com.br/2014/03/controle-de-dcnt.html>. Acesso em: 30 set. 2014.

${ }^{2}$ BIBLIOTECA dos Prêmios Nobel de Literatura. Patrocinada pela Academia Sueca e pela Fundação Nobel. Prêmio de 1953. Winston Churchill (Inglaterra). Rio de Janeiro: Opera Mundi, 1973.
} 
reabilitação; (7) identificação de fatores de risco isolados; (8) prevenção primária de fatores de risco isolados; (9) prevenção primordial; (10) identificação de suscetibilidade genética; (11) identificação de fatores de risco associados e comuns a várias DCNT; (12) advocacia; (13) educação para a saúde; (14) abordagem associada dos fatores de risco e das DCNT em escala populacional; (15) promoção da saúde; (16) estilos de vida; (17) saúde global; (18) estudo de mecanismos neurais do comportamento e da dependência; (19) psicologia social; (20) abordagem das causas; (21) implicações macroeconômicas; (22) determinação social das doenças; (23) componentes emocionais: autoestima e rejeição; e (24) saúde urbana, ambiente e equidade.

Em 8 de setembro de 2000, a 55ª Assembleia Geral da Organização das Nações Unidas (ONU) adotou uma resolução chamada Declaração do Milênio ${ }^{3}$, voltada para:

um mundo pacífico, próspero e justo, reconhecendo as responsabilidades em sustentar princípios de dignidade humana, igualdade e equidade e a obrigação com toda a população mundial, especialmente os mais vulneráveis e, em particular, as crianças do mundo a quem o futuro pertence.

Reafirmam-se a importância do desenvolvimento humano e a necessidade do combate à pobreza e à falta de educação, propondo-se até 2015 reduzir pela metade a proporção dos que vivem com menos de um dólar por dia, sofrem fome e não têm água segura para beber. Dentre os Objetivos do Milênio (OM), não se menciona diretamente nenhuma das DCNT, na época responsáveis por $63 \%$ da mortalidade mundial, com incidência mais precoce nas populações mais pobres e com o agravante de contribuírem, em círculo vicioso, para a manutenção do subdesenvolvimento.

Mesmo sem menção específica, ao serem alcançados os OM, deverá haver benefícios em relação às doenças não contempladas diretamente, de acordo com a teoria da programação fetal das doenças do adulto e modificações epigenéticas da expressão dos genes (Teoria de Barker) ${ }^{4}$. As DCNT, mesmo não constantes nos OM, serão controladas indiretamente com a melhoria das condições de desenvolvimento fetal e nos primeiros anos de vida, garantindo segurança alimentar, melhorando a saúde e as condições de vida das mães, promovendo a educação e reduzindo o peso negativo da pobreza que destrói a autoestima, favorece os fatores de risco e dificulta o acesso a melhor assistência.

Em setembro de 2011, a ONU promoveu em Nova Iorque outra Reunião de Alto Nível para rediscutir os OM, resultando em nova resolução adotada pela

\footnotetext{
${ }^{3}$ Declaração e objetivos do milênio. UNITED NATIONS. Resolution adopted by the General Assembly. United Nations. 55/2 Millennium Declaration. Disponível em: <http://www.un.org/millennium/declaration/ares552e.pdf>. Acesso em: 30 set. 2014.

${ }^{4}$ THE BARKER Theory. Disponível em: <http://www.thebarkertheory.org/index.php>. Acesso em: 30 set. 2014.
} 
Assembleia Geral (66/2) em que consta uma Declaração Política sobre Prevenção e Controle de DCNT $T^{5}$. Seguindo-se ao encontro de líderes mundiais (entre os quais estava a atual Presidente da República do Brasil, Dilma Roussef), foi elaborado um plano de ação para 2013-2020 .

\section{Perspectiva nacional}

No início da década de 1970, realizou-se no Estado do Rio Grande do Sul uma análise institucional da Secretaria da Saúde e do Meio Ambiente (SSMA), em cujo grupo sobre epidemiologia cardiovascular participou este autor. Depois de se examinarem as informações disponíveis, chegou-se à conclusão de que elas eram muito pobres ou inexistentes (para fundamentar a discussão do tema, foram utilizados dados americanos) e de que não existia nenhum programa de saúde pública relacionado com as DCNT, embora houvesse indícios da importância do problema do ponto de vista de saúde pública ${ }^{7}$.

Em artigo publicado no periódico Chronicle of the World Health Organization, da Organização Mundial da Saúde (OMS) ${ }^{8}$, Thomas Strasser abordou a prevenção comunitária da febre reumática em países com desenvolvimento social e econômico equiparável ao do Brasil. O texto serviu como inspiração para se propor iniciativa semelhante por aqui, seguindo modelo proposto para populações do outro lado do oceano.

Ao solicitarmos autorização a Daniel Lopes Ferrer - então gerente do escritório da Organização Pan-Americana da Saúde (OPAS) no Estado do Rio Grande do Sul - para utilizarmos o protocolo da OMS, a informação sobre nosso projeto na Secretaria de Saúde foi repassada para Washington, onde Jorge Litvak (endocrinologista chileno) recém-assumira posição com propósitos de promover programas comunitários para estudo e controle de DCNT em países do continente ${ }^{9}$. Em 1975, a cidade de Porto Alegre sediou um primeiro encontro de representantes de alguns países para desenvolver estudos epidemiológicos e prevenção secundária da febre reumática no Brasil, Argentina, Paraguai, Chile, Bolívia, Peru, Venezuela e Guatemala ${ }^{10}$. Muitos outros assessores da OPAS e

5UNITED NATIONS. Resolution adopted by the General Assembly. 66/2 Political Declaration of the High-level Meeting of the General Assembly on the Prevention and Control of Non-communicable Diseases. Disponivel em: <http:// www.who.int/nmh/events/un_ncd_summit2011/political_declaration_en.pdf>. Acesso em: 30 set. 2014.

${ }^{6}$ WORLD HEALTH ORGANIZATION - WHO. Global action plan for the prevention and control of NCDs 2013-2020. Disponivel em: <http://www.who.int/nmh/publications/ncd-action-plan/en/>. Acesso em: 30 set. 2014.

${ }^{7}$ ANÁLISE Institucional e programação de atividades 1972-74. Porto Alegre: Secretaria da Saúde, Governo do Estado do RS, 1972.

${ }^{8}$ WORLD HEALTH ORGANIZATION - WHO. Expert Consultation on Rheumatic Fever and Rheumatic Heart Disease. Rheumatic fever and rheumatic heart disease: report of a WHO Expert Consultation. Geneva, Switzerland, 29 October - 1 November 2001. (WHO technical report series ; 923). Disponível em: <http://www. who.int/cardiovascular_diseases/resources/en/cvd_trs923.pdf>. Acesso em: 30 set. 2014.

${ }^{9}$ OPS: Plan Decenal de Salud para las Americas. III Reunion Especial de Ministros de Salud. Oct. 1972.

${ }^{10}$ PREVENCIÓN y control de la fiebre reumática en la comunidad. Washington, DC, Organización Panamericana de la Salud, 1980. Nova edição em Inglês: PREVENTION and Control of Rheumatic Fever in the Community. Washington, DC, Organización Panamericana de la Salud, 1985. (PAHO Scientific Publication No. 399). 
da OMS deveriam ser citados pelo papel relevante exercido no desenvolvimento inicial e na promoção das estratégias para o controle das DCNT. Entre eles, destacam-se: Luis Ruis, Hector Boffi Boggero, Antonio Menna, Helena Restrepo, Sigfried Böttig, Ivan Gyarfas, Shanti Mendes e Porfírio Nordet.

A presença de um grupo excepcional de sanitaristas no Conselho da SSMA do Estado - Valdir Arcoverde, Moacyr Scliar, Clovis Tigre, Cláudio Silveira, Ayrton Fischmann, Jorge Ossanai, Ciro de Quadros, Germano Bonow - e no Serviço de Doenças Crônicas - Maria Inês Reinert Azambuja, Sérgio Luiz Bassanesi, Maria Helena Engel Rosito, Maryleda Costa, Ana Maria Britto e José Baldi - certamente foi determinante para aceitação e desenvolvimento da proposta na agenda de saúde pública do Rio Grande do Sul.

A experiência logo propiciou sua adaptação e extensão para a abordagem de hipertensão arterial, tabagismo, sedentarismo, diabetes mellitus, obesidade, câncer ginecológico e doença de Chagas, chamando atenção do Ministério da Saúde e de outros estados brasileiros e motivando extenso e profícuo intercâmbio. Em 1977, o Ministério da Saúde convocou no Rio de Janeiro uma reunião coordenada por Humberto Torloni (então dirigente do Instituto Nacional do Câncer, único organismo voltado para assistência a problemas do conjunto DCNT). À reunião compareceram Rubens Maciel (egresso de estágio na OPAS), Cláudio Penna, Romero Bezerra Barbosa, Eduardo de Azeredo Costa e outros profissionais da saúde (entre os quais este autor), representando a experiência gaúcha. Em seguida foi criado o Departamento de Doenças Crônicas Não Transmissíveis no Ministério da Saúde, coordenado por Edmur Pastorello, Geniberto Paiva Campos, Romero Barbosa, Ely Toscano Barbosa e outros.

Com a coordenação de Eduardo Azeredo Costa e deste autor e com a orientação de Geoffrey Rose ${ }^{11}$, realizou-se em 1978 uma pesquisa populacional sobre pressão arterial e outros fatores de risco, aleatória, estratificada e representativa da população adulta (de 20 a 74 anos) do Rio Grande do Sul. Na época, foi o estudo mais extenso relacionado a essa temática em países com característica de desenvolvimento humano semelhante ao do Brasil ${ }^{12}$.

Seguindo essa mesma linha, dentro de um referencial estratégico, nos últimos 30 anos diversas oportunidades de pesquisa e ação surgiram e foram sendo estrategicamente aproveitadas, das quais algumas podem ser destacadas (sendo o controle do tabagismo a de maior impacto):

\footnotetext{
${ }^{11}$ ESTRATÉGIAS Preventivas: o caso do amianto no Brasil. Disponível em: <http://eduardodazeredocosta. blogspot.com.br/2013/12/estrategias-preventivas-o-caso-do.html>. Acesso em: 30 set. 2014.

${ }^{12}$ COSTA, E. Sal e a pressão arterial na população adulta do Estado do RS, Brasil. 1980. Tese (Pós-doutorado), London School of Hygiene and Tropical Diseases. Londres, 1980.
} 
- Em 1980, estudo de prevalência de uso do tabaco e conhecimentos básicos sobre tabagismo na população escolar de Porto Alegre e do Rio Grande do Sul, acompanhado de elaboração de material educativo para professores, população-alvo e intervenção no processo educacional ${ }^{13}$.

- Technical Report on Smoking in Developing Countries, da Organização Mundial da Saúde, $1982^{14}$.

- Censo Nacional de Prevalência do Diabetes Mellitus, 1988.

- Em 1987, estudo populacional de monitorização de fatores de risco (Estudo MORE) realizado em capitais latino-americanas (Porto Alegre, São Paulo, Havana, Bogotá, Caracas e Santiago) e mais duas cidades no México, na fronteira com os Estados Unidos ${ }^{15}$.

- Estudo do Banco Mundial coordenado por John Briscoe sobre o novo desafio da saúde do adulto ${ }^{16}$.

- Em 1992, Surgeon General Report: Smoking in the Americas ${ }^{17}$.

- Relatório de Desenvolvimento do Banco Mundial de 1993, em que foi apresentada nova forma de contabilizar o impacto das doenças por anos de saúde perdidos e ajustados ${ }^{18}$.

- Rheumatic Fever Prevention in Developing Countries Joint Programme WHO/ UNESCO/WHF, Genebra, $1994^{19}$.

- White Book "Impending global pandemic of cardiovascular diseases: challenges and opportunities for the prevention and control of cardiovascular diseases in developing countries and economies in transition", da World Heart Federation ${ }^{20}$.

${ }^{13}$ CONTROL del habito de fumar - Projeto Fumo. Taller Subregional para el Cono Sur y Brasil. Cuaderno Técnico. 2, OPS, 1986.

${ }^{14}$ WORLD HEALTH ORGANIZATION - WHO. Smoking control strategies in developing countries. Geneva, 1983. (Technical Report Series 695).

${ }^{15}$ ACHUTTI, A.; VICTORA, C. Promoção/Proteção da Saúde. Relatório de atividades realizadas no decurso do ano. Faculdade de Medicina da UFRGS e Programa Saúde do Adulto, OPAS. Porto Alegre 1988. Mimeo.

${ }^{16}$ BRISCOE, J. Brazil: the new challenge of adult health: a World Bank Country Study. Washington, DC., The World Bank, 1990.

${ }^{17}$ U.S. DEPARTMENT OF HEALTH AND HUMAN SERVICES. Smoking and Health in the Americas. Atlanta, Georgia: U.S. Department of Health and Human Services, Public Health Service, Centers for Disease Control, National Center for Chronic Disease Prevention and Health Promotion, Office on Smoking and Health, 1992; DHHS Publication No. (CDC) 92-8419. Disponível em: <http://profiles.nlm.nih.gov/ps/access/NNBBBJ.pdf>. Acesso em: 30 set. 2014.

${ }^{18}$ INVESTING in Health World Development Report 1993. Oxford: Oxford University Press; World Bank, 1993.

${ }^{19}$ WORLD HEALTH ORGANIZATION - WHO. Reporto $f$ a Consultation to review Progress and develop Future activities - Geneva, 29 November - 1 December 1999. Capítulo II. Disponível em: <http://whqlibdoc.who. int/hq/2000/WHO_CVD_00.1.pdf>. Acesso em: 30 set. 2014.

${ }^{20} \mathrm{CHOCKALINGAM,} \mathrm{A.;} \mathrm{BALAGUER-VINTRO,} \mathrm{I.;} \mathrm{ACHUTTI,} \mathrm{A.;} \mathrm{DE} \mathrm{LUNA,} \mathrm{A.B.;} \mathrm{CHALMERS,} \mathrm{J.;} \mathrm{FARINARO,} \mathrm{E.;} \mathrm{LAU-}$ ZON, R.; MARTIN, I.; PAPP, J.G.; POSTIGLIONE, A.; REDDY, K.S.; TSE, T.F. The World Heart Federation's white book: impending global pandemic of cardiovascular diseases: challenges and opportunities for the prevention and control of cardiovascular diseases in developing countries and economies in transition. Canadian Journal of Cardiology, Montreal v. 16, n. 2, p. 227-229, Feb. 2000. PMID: 10694594. 
- Em 1997, em seguimento ao I Seminário Nacional sobre Epidemiologia e Prevenção de Doenças Cardiovasculares, realizado em Gramado, criou-se a rede virtual Amicor ${ }^{21}$, com ênfase em DCNT e outros fatores que de alguma forma podem estar relacionados com as causas dessas doenças.

- Em 1997, por iniciativa do Professor Bernard Lown (Boston), foi criada a rede ProCOR, com algumas características semelhantes ${ }^{22}$.

- Norma técnica sobre Prevenção do Tabagismo, Sociedade Brasileira de Cardiologia, 2013.

\section{Tópicos que merecem mais discussão}

Cada tópico que segue poderia ser o título para um novo artigo ou mesmo para um debate. Na dimensão deste texto, eles são apresentados como uma provocação, com possibilidade de suscitar mais estudos e reflexões.

\section{Transposição de experiências}

Sem negar a importância da troca de experiências, observa-se sempre a tentação de replicar práticas de sucesso obtidas com uma população em outra, $o$ que, entretanto, pode ter limitações, mesmo dentro de um mesmo país ou de uma mesma cidade, considerando-se estratos sociais e culturas distintos. É emblemático o que ocorreu por aqui quando, com a maior boa vontade, o saudoso chargista Ziraldo elaborou um cartaz de um homem todo adornado com colares e pulseiras chamativas e fumando, com a frase: "Fumar é cafona". Para os especialistas em saúde, fumar realmente significava cafonice, porém, para a população das comunidades do Rio de Janeiro, fumar era sinal de sucesso e objeto de desejo.

\section{Meia informação ou generalização indevida}

"Sou 12 por 8 ". Este slogan, intensivamente utilizado como alerta para a detecção precoce da hipertensão arterial, pode ser questionado, pois, se para indivíduos jovens, tranquilos e em repouso trate-se de níveis desejáveis, parte da população - principalmente os mais idosos cujas artérias têm elasticidade naturalmente reduzida - não consegue satisfazer o critério e até pode ser inconveniente que procure atingi-lo, aumentando a dose da medicação. Essa frustração pode ser motivo de mais ansiedade e até funcionar como em um círculo vicioso. $\mathrm{O}$ uso da publicidade simplista discrimina e causa malefícios para quem não pode ou não deve ser enquadrado em certos critérios.

\footnotetext{
${ }^{21}$ AMICOR. Disponível em: <http://amicor.blogspot.com>. Acesso em: 30 set. 2014.

${ }^{22}$ Iniciativa do Professor Bernard Lown (1997) Rede PoCOR.
} 


\section{Recomendações indiscriminadas}

Não se pode comer a gema do ovo, gorduras saturadas devem ser evitadas e é preciso reduzir todo tipo de gorduras, a ingestão de sal e o consumo de açúcar. Todas essas recomendações, para parte da população, podem não ter a mesma importância que têm para os especialistas em saúde.

\section{Redução de danos}

Frente à dificuldade de atingir objetivos ideais, em algumas situações, como no tabagismo, preconiza-se a redução do dano, mais recentemente com utilização de cigarro eletrônico. Embora com esse recurso seja possível evitar a contaminação de circunstantes (fumo secundário) e reduzir algumas substâncias produzidas na combustão do tabaco (como alcatrões), o abandono da dependência fica dificultado pela manutenção da substância química básica responsável por ela, bem como pelo ritual da utilização dos lábios e da baforada. Provavelmente a indústria tenha interesse no cigarro eletrônico, pelo fato de o produto final ter maior valor agregado e funcionar como uma ilusão de sucesso ou, ainda, como uma novidade.

\section{Demanda espontânea $x$ rastreamento de casos}

Podem existir controvérsias relacionadas com a assistência frente a doenças não contagiosas, entre apenas informar e criar condições para um diagnóstico adequado ou buscar ativamente casos na comunidade.

\section{Exagero promocional}

O entusiasmo ou a impulsividade dos promotores e educadores para a saúde os leva, às vezes, a agir com o fanatismo de ativistas políticos, interferindo sem paciência ou sem compreensão do processo de dependência em fatores comportamentais.

\section{Multicausalidade}

Os esforços utilizados na modificação do comportamento podem causar estresse e a substituição de um fator de risco por outro ou vários outros.

\section{Discriminação}

A identificação de um fator de risco - como tabagismo, obesidade e outras dependências - frequentemente funciona como fator de minus valia, com significado não somente para outras pessoas e profissionais da saúde, como também para o próprio indivíduo, criando motivo a mais para frustração, estresse e sofrimento. 


\section{Descrédito por interpretações viciosas}

O exagero na veiculação de informações, índices e interpretações pode funcionar como elemento de descrédito em relação aos profissionais da saúde, particularmente por parte dos indivíduos que mais precisam de ajuda.

\section{Dificuldades na monitorização}

Muitos dos indicadores de interesse para uma adequada monitorização do comportamento e das prevalências podem ficar ocultos ou fogem ao controle oficial, como é o caso do contrabando de cigarros, álcool e outras drogas. Sua estigmatização colabora com a tendência de eles escaparem da vista.

Embora benéficas por um lado, as restrições legais funcionam como fomento ao comércio ilegal, como estímulo ao tráfico e à demanda e como causa da supervalorização do produto.

\section{Especialização e atenção primária}

Não pode existir conflito entre essas duas etapas da assistência, mas existe competição por recursos e por valorização na cultura médica. A especialização desenvolve-se com vantagens, mas facilmente se distancia da perspectiva populacional, da prevenção e do controle.

\section{Visão antropológica}

Em geral, na formação médica constata-se um reduzido conhecimento sobre as bases antropológicas do comportamento humano: identificação grupal, adesão a modelos como argumentos de modismo, discriminação e abuso.

\section{Etapas de evolução do comportamento}

São bem conhecidos os princípios que regem o comportamento, mas não custa relembrar: o primeiro passo é o da informação gerando conhecimento; depois vem uma fase de contemplação de incorporação ou mudança, à qual se segue a da formação de uma atitude favorável, embora ainda exigindo esforço; e, por fim, a fase da mudança, quando o comportamento se incorpora à cultura grupal e pode ser quase insensivelmente copiado sem maior esforço.

Não se pode esquecer de que, em geral, o contato com a realidade se faz primeiro por via emocional, pela qual é possível sentir mesmo sem compreender e já criar uma predisposição mesmo sem o conhecimento.

\section{Circuito da recompensa}

Prazer imediato e fuga da dor são mecanismos baseados em estruturas cerebrais ligadas à sobrevivência, não somente humana, que podem ajudar a 
compreender e buscar o controle do comportamento, visando à intervenção sobre fatores comportamentais e sua relação com as DCNT.

\section{Processo decisório}

Também é preciso reconhecer as deficiências no conhecimento para melhor compreender e lidar com o processo decisório, do ponto de vista tanto do indivíduo como grupal. A indústria e os profissionais de marketing em geral estão mais bem atualizados quanto aos caminhos e a probabilidade de sucesso nas negociações e decisões. Médicos ingenuamente acreditam serem suficientes a racionalidade e as evidências científicas, enquanto economistas e psicólogos têm lidado mais adequadamente com a motivação das escolhas e tomadas de decisões.

\section{Estilo de vida}

Juntamente com a valorização do controle dos fatores de risco, a expressão estilo de vida adquiriu força de slogan promocional, desviando a atenção sobre a importância da determinação social das doenças, como se o estilo fosse uma espécie de escolha do indivíduo, minimizando outras forças determinantes do comportamento humano.

\section{Cooperação internacional}

Certamente a troca de experiências e o intercâmbio transcultural, que têm um papel importante na disseminação (poder-se-ia dizer na transmissão ou difusão) de padrões de comportamento de risco, também são fundamentais na inspiração e no suporte a iniciativas que visam ao controle das DCNT.

A cooperação tem ficado mais facilitada com o progresso dos meios de comunicação eletrônicos, capazes de manter quem quiser em reunião permanente.

Em 1997 (em seguimento ao I Seminário Nacional sobre Epidemiologia e Prevenção de Doenças Cardiovasculares, em Gramado), iniciou-se a rede chamada Amicor, com ênfase em DCNT e em situações que, de alguma forma, podem estar relacionadas às causas dessas doenças. Poucos meses depois, o professor Bernard Lown (Boston) deu início à rede ProCOR, com algumas características semelhantes.

\section{Custo social}

Não tem sido suficientemente avaliado o custo social dos fatores de risco e da morbimortalidade precoce, como gastos com investigação e tratamento; sofrimento do indivíduo, da família e do grupo; perda de produtividade e aposentadoria precoce. O que existe não está em linguagem convincente para políticos e empresários. 


\section{Coexistência com interesses secundários}

Todo esforço no sentido de controle dos fatores de risco coexiste com outros interesses - como propaganda e promoção de produtos e comportamentos de risco -, em geral promovidos por fontes bem mais poderosas do ponto de vista econômico, social e político. Vale reproduzir um parágrafo de Geoffrey Rose: "Nós temos uma responsabilidade pessoal com a prevenção, tanto na pesquisa como na prática médica. Quando os médicos não aceitam essa responsabilidade, a prevenção é assaltada por propagandistas acríticos, por impostores e por interesses comerciais escusos"23.

\section{Dependência da taxação}

Se o imposto é um dos recursos para elevar o preço do produto que causa dependência visando à redução no consumo, é preciso levar em conta que o governo torna-se dependente desses recursos e pode ficar ambivalente sobre medidas que possam estancar essa fonte. $\mathrm{O}$ crescimento das taxas impulsiona também o poder das companhias interessadas, e não é de surpreender que elas mesmas em geral sejam favoráveis ao aumento da taxação. O tema deste ano para o Dia Mundial sem Tabaco é justamente o aumento dos impostos visando a reduzir o consumo. Para os mais dependentes, pouco importa o aumento do preço do produto.

\section{Solidariedade global}

No caso do Brasil, grande parte da produção de tabaco é destinada à exportação e consumida fora do país, argumento utilizado a favor do protecionismo de nossas lavouras de fumo. Falta-nos a consciência de uma solidariedade global, como falta ao contrabandista e ao traficante.

\section{Doença social}

A necessidade do uso de substâncias e artifícios causadores de dependência precisa ser examinada à luz do tipo de sociedade que construímos e na qual vivemos, onde um grande número de pessoas se vale de expedientes desse tipo para buscar recompensa e atenuar o sofrimento causado pela desigualdade, pelo desequilíbrio e pelas frustrações.

\section{Fronteira entre doenças transmissíveis e doenças não transmissíveis}

Fica cada vez mais claro que a dicotomia das doenças que deu origem à designação de DCNT não auxilia na compreensão da etiopatogenia das

\footnotetext{
${ }^{23}$ ROSE, Geoffrey. Strategy of prevention: lessons from cardiovascular disease. Disponivel em: <http://www. ncbi.nlm.nih.gov/pmc/articles/PMC1506445/pdf/bmjcred00661-0031.pdf>. Acesso em: 30 set. 2014.
} 
mesmas, pois cada vez mais se encontram micro-organismos ligados a DCNT. E, no sentido contrário, também aumenta a suscetibilidade às doenças transmissíveis e prejudica a evolução em relação a elas. O sistema imunitário e as reações inflamatórias têm contribuído para acrescentar uma visão intrigante dentro do paradigma médico convencional ${ }^{24}$.

\section{Saúde urbana}

Sob uma perspectiva mais ampla, o referencial da saúde urbana - os centros urbanos são concretamente onde a população nasce, cresce, reproduz-se, produz e morre - ligado às estruturas de poder é mais forte do que a capacidade individual de resistir ${ }^{25}$.

\section{Ambiente}

Para além do óbvio da não destruição do próprio patrimônio material, natural e cultural, com consequência imediata e para as gerações futuras, é preciso discutir os para-efeitos, às vezes inadvertidos, causados pelo próprio tratamento (pesticidas, drogas, hormônios, antibióticos, psicotrópicos e outros produtos químicos) da água e dos alimentos promovido como preventivo de DCNT.

A poluição ambiental pode ser vista para além da área químico-física e inclui poluição visual, radiações, , ambiente doméstico e laboral e equipamento urbano de deslocamento e lazer. $\mathrm{O}$ ambiente psicológico, a agressividade, a competitividade, a corrupção, a insegurança, a desconfiança, a falta de perspectiva de vida, a falta de solidariedade e o estresse social também são fatores ambientais.

\section{Desigualdade}

Em todos os tópicos delineados aqui, pode-se ver a iniquidade social e valorizar a determinação social de saúde/doença em que se salientam as DCNT, conforme tem defendido Michael Gideon Marmot por meio principalmente da comissão sobre determinantes sociais da $\mathrm{OMS}^{26}$. Entenda-se que, quando se fala em desigualdade, não se está limitando à desigualdade econômica e de condições materiais de vida, mas também se está referindo ao tratamento desigual, à discriminação, ao prejuízo da autoestima e à falta de perspectiva de vida.

\footnotetext{
${ }^{24}$ AZAMBUJA, Maria Inês Reinert; ACHUTTI, A.; LEVINS, Richard. The inflammation paradigm: towards a consensus to explain coronary heart disease mortality in the $20^{\text {th }}$ century. CVD Prevention and Control, v. 3, p. 69-76, 2008.

${ }^{25}$ AZAMBUJA, Maria Inês Reinert et al. Saúde urbana, ambiente e desigualdades. Revista Brasileira de Medicina da Família e Comunidade, Rio de Janeiro v. 6, n. 19, 2011. Disponível em: <http://rbmfc.org.br/rbmfc/article/view/151/298.\%20artigo\%20em\%20colabora\%C3\%A7\%C3\%A3o>. Acesso em: 30 set. 2014.

${ }^{26}$ MARMOT, M.G. Social determinants of health. Disponivel em: <http://www.who.int/social_determinants/ publications/en/>. Acesso em: 30 set. 2014.
} 


\section{Considerações finais}

1. Diferentemente do que ocorreu com as doenças transmissíveis, apesar de todos os sucessos e evidências, não se percebe a incorporação efetiva de uma perspectiva sanitária entre os especialistas dedicados à assistência das DCNT.

2. Assim como aconteceu com as doenças transmissíveis, em que as ações efetivas foram dirigidas às causas da disseminação dos germes, não se percebe face às DCNT uma atitude que se antecipe aos fatores de risco, relacionada com o desenvolvimento humano e com a distribuição do poder e da riqueza na sociedade.

3. O desenvolvimento de programas preventivos ainda é voltado para fatores de risco isolados, como se o comportamento humano fosse totalmente autônomo, e não dependente de estruturas sociais que dominam a cultura.

4. Os sucessos da ciência e tecnologia no tratamento heroico na fase avançada das DCNT distraem a atenção das mudanças necessárias e reduzem a capacidade de investimentos na organização de cidades com equipamentos que favoreçam a qualidade de vida e que possam se desenvolver mais justas, produtivas e pacíficas.

5. Faltam estudos e demonstrações que convençam políticos e empresários de que, como aconteceu com o saneamento básico, a redução da pobreza, a melhora da educação e dos níveis de emprego, o acesso à moradia, ao transporte e ao lazer, a segurança e a perspectiva de vida podem ter grande impacto no controle das DCNT.

6. A atual etapa de desenvolvimento social global conta com facilidades tecnológicas de conectividade e comunicação que estão facilitando a troca de informação e influindo muito rapidamente no comportamento (fatores de risco comportamentais) e em sua disseminação (assim como a proximidade entre as pessoas e a falta de cuidados higiênicos facilitavam a transmissão dos germes). Parecem necessárias uma atualização e uma melhor utilização dos recursos de controle.

7. Face ao profundo desencontro entre o paradigma dominante na sociedade atual e os valores necessários para se conseguir desenvolvimento sustentado no controle efetivo das DCNT, faz-se necessário mobilizar todos os recursos de comunicação e articulação para se conseguir progressiva aproximação da revolução cultural necessária.

8. Embora exista muito discurso em torno do controle das DCNT e haja uma progressiva consciência de sua importância, é muito provável que o impacto mensurável não se deva tanto a intervenções específicas, mas ao controle de algumas variáveis do desenvolvimento humano, assim como ocorreu com as doenças transmissíveis. Entretanto, a persistência de uma cultura de risco, da violência, do preconceito e da desigualdade é uma evidência que aponta para o longo caminho ainda a percorrer e para as grandes possibilidades futuras ${ }^{27}$.

\footnotetext{
${ }^{27}$ HORTON, Richard. A new vision for the post 2015 era. The Lancet, v. 382, n. 9897, p. 1012, 21 Sept. 2013. doi:10.1016/S0140-6736(13)61936.
} 


\section{Referências}

ACHUTTI, A. Cardiovascular prevention: a global challenge. Tobacco e alcohol control: avoidable risk factor of CVDs. Disponível em: <http://jornal.cardiol.br/2013/novembro/ edicao/\#VI>. Acesso em: 30 set. 2014.

ACHUTTI, A. De médicos e economia. Jornal ZH, 16 out. 2013. Disponível em: <http:// amicor.blogspot.com.br/2013/10/de-medicos-e-economia.html>. Acesso em: 30 set. 2014.

ACHUTTI, A. Prevention of cardiovascular diseases and the promotion of health. Ciência \& Saude Coletiva, Rio de Janeiro v. 17, n. 1, p. 18-20, jan. 2012.

ACHUTTI, A.; VICTORA, C. Promoção/Proteção da Saúde. Relatório de atividades realizadas no decurso do ano. Faculdade de Medicina da UFRGS e Programa Saúde do Adulto, OPAS. Porto Alegre 1988. Mimeo.

AMICOR. Disponível em: <http://amicor.blogspot.com.br/2014/03/controle-de-dcnt.html >. Acesso em: 30 set. 2014.

AMICOR. Disponível em: <http://amicor.blogspot.com>. Acesso em: 30 set. 2014.

ANÁLISE Institucional e programação de atividades 1972-74. Porto Alegre: Secretaria da Saúde, Governo do Estado do RS, 1972.

AZAMBUJA, Maria Inês Reinert et al. Saúde urbana, ambiente e desigualdades. Revista Brasileira de Medicina de Família e Comunidade, Rio de Janeiro v. 6, n. 19, 2011. Disponível em: <http://rbmfc.org.br/rbmfc/article/view/151/298.\%20artigo\%20em\%20 colabora\%C3\%A7\%C3\%A3o >. Acesso em: 30 set. 2014.

AZAMBUJA, Maria Inês Reinert; ACHUTTI, A.; LEVINS, Richard. The inflammation paradigm: towards a consensus to explain coronary heart disease mortality in the $20^{\text {th }}$ century. CVD Prevention and Control, v. 3, p. 69-76, 2008.

BIBLIOTECA dos Prêmios Nobel de Literatura. Patrocinada pela Academia Sueca e pela Fundação Nobel. Prêmio de 1953. Winston Churchill (Inglaterra). Rio de Janeiro: Opera Mundi, 1973.

BRISCOE, J. Brazil: the new challenge of adult health: a World Bank Country Study. Washington, DC., The World Bank, 1990.

CHOCKAlingam, A.; BAlAgUER-VINTRO, I.; ACHUTTI, A.; DE LUNA, A.B.; CHALMERS, J.; FARINARO, E.; LAUZON, R.; MARTIN, I.; PAPP, J.G.; POSTIGLIONE, A.; REDDY, K.S.; TSE, T.F. The World Heart Federation's white book: impending global pandemic of cardiovascular diseases: challenges and opportunities for the prevention and control of cardiovascular diseases in developing countries and economies in transition. Canadian Journal of Cardiology, Montreal v. 16, n. 2, p. 227-229, Feb. 2000. PMID: 10694594.

CONTROL del habito de fumar - Projeto Fumo. Taller Subregional para el Cono Sur y Brasil. Cuaderno Técnico. 2, OPS, 1986. 
COSTA, E. Sal e a pressão arterial na população adulta do Estado do RS, Brasil. 1980. Tese (Pós-doutorado), London School of Hygiene and Tropical Diseases. Londres, 1980.

ESTRATÉGIAS Preventivas: o caso do amianto no Brasil. Disponível em: <http:// eduardodazeredocosta.blogspot.com.br/2013/12/estrategias-preventivas-o-caso-do.html >. Acesso em: 30 set. 2014.

HORTON, Richard. A new vision for the post 2015 era. The Lancet, v. 382, n. 9897, p. 1012, 21 Sept. 2013. doi:10.1016/S0140-6736(13)61936.

I DIRETRIZ Brasileira de Prevenção Cardiovascular. Arquivos Brasileiros de Cardiologia, Rio de Janeiro, v. 101, n. 6, supl. 2, dez. 2013.

INVESTING in Health World Development Report 1993. Oxford: Oxford University Press; World Bank, 1993.

MARMOT, M.G. Social determinants of health. Disponível em: <http://www.who.int/ social_determinants/publications/en/>. Acesso em: 30 set. 2014.

OPS: Plan Decenal de Salud para las Americas. III Reunion Especial de Ministros de Salud. Oct. 1972.

PREVENCIÓN y control de la fiebre reumática en la comunidad. Washington, DC, Organización Panamericana de la Salud, 1980.

PREVENTION and Control of Rheumatic Fever in the Community. Washington, DC, Organización Panamericana de la Salud, 1985. (PAHO Scientific Publication No. 399).

ROSE, Geoffrey. Strategy of prevention: lessons from cardiovascular disease. Disponível em: $<$ http://www.ncbi.nlm.nih.gov/pmc/articles/PMC1506445/pdf/bmjcred00661-0031.pdf>. Acesso em: 30 set. 2014.

THE BARKER Theory. Disponível em: <http://www.thebarkertheory.org/index.php $>$. Acesso em: 30 set. 2014.

U.S. DEPARTMENT OF HEALTH AND HUMAN SERVICES. Smoking and Health in the Americas. Atlanta, Georgia: U.S. Department of Health and Human Services, Public Health Service, Centers for Disease Control, National Center for Chronic Disease Prevention and Health Promotion, Office on Smoking and Health, 1992; DHHS Publication No. (CDC) 92-8419. Disponível em: <http://profiles.nlm.nih.gov/ps/access/NNBBBJ.pdf > Acesso em: 30 set. 2014.

WORLD HEALTH ORGANIZATION - WHO. Expert Consultation on Rheumatic Fever and Rheumatic Heart Disease. Rheumatic fever and rheumatic heart disease: report of a WHO Expert Consultation. Geneva, Switzerland, 29 October - 1 November 2001. (WHO technical report series ; 923). Disponível em: <http://www.who.int/cardiovascular_diseases/resources/ en/cvd_trs923.pdf $>$. Acesso em: 30 set. 2014. 
WORLD HEALTH ORGANIZATION - WHO. Global action plan for the prevention and control of NCDs 2013-2020. Disponível em: <http://www.who.int/nmh/publications/ncdaction-plan/en/>. Acesso em: 30 set. 2014.

Report of a consultation to review progress and develop future activites - Geneva, 29 November - 1 December 1999. Disponível em: <http://whqlibdoc.who.int/hq/2000/ WHO_CVD_00.1.pdf>. Acesso em: 30 set. 2014.

Smoking control strategies in developing countries. Geneva, 1983. (Technical Report Series 695).

Aloyzio Achutti - Professor aposentado da Universidade Federal do Rio Grande do Sul. Consultor da Organização Pan-Americana da Saúde e da Organização Mundial da Saúde. Médico. Porto Alegre/RS, Brasil.E-mail: achutti@gmail.com. 